\title{
Strømgren and $\mathrm{H} \beta$ photometry of $\mathrm{OB}$ stars in the region of the Carina Spiral Feature
}

\author{
N.T. Kaltcheva \\ Department of Astronomy, University of Sofia "St. Kliment Ohridski", 5 James Bourchier Avenue, 1126 Sofia, Bulgaria \\ e-mail: nadia@phys.uni-sofia.bg
}

Received 17 December, 1996; accepted 5 May, 1997

\begin{abstract}
Strømgren and $\mathrm{H} \beta$ photometry of 130 stars in the Carina section of the Milky Way is presented. The color excesses and the distances of all stars are obtained. A good agreement is found between the CM and HR diagrams and the spatial distribution of these stars.
\end{abstract}

Key words: stars: early-type — stars: distances galaxy: structure — stars: HR diagram

\section{Introduction}

The region between $l=284^{\circ}-294^{\circ}$ galactic longitude in Carina has long been known to be rich in highly luminous and massive stars. The objective prism survey of Graham \& Lynga (1965) revealed about 450 non-cluster OB-type stars in this part of the sky, and this number increased with the publication of the "Luminous Stars in the Southern Milky Way" catalog by Stephenson \& Sanduleak (1971). In that part of the Galaxy, the interstellar absorption is evidently small, and since the Carina section is in a direction along a spiral arm, an extremely large young structure can be seen on the line of sight. The clumping of these OB stars into associations and also the connection of possible associations with young open clusters situated in this field is widely discussed. In the Humphreys (1978) catalog, two associations are separated in this direction: Car OB1 at $l=284.2^{\circ}-288.3^{\circ}$ and Car OB2 at $l=289^{\circ}-291.2^{\circ}$, respectively. Nearly 460 noncluster stars from the compact early-type group Car OB2 in the direction $l=290^{\circ}, b=0.4^{\circ}$ in a square with approximate dimensions 30 arcmin have been studied photographically by Seggewiss (1970) and photoelectrically by Garcia (1994) in the $U B V$ system. Kaltcheva \& Georgiev

Send offprint requests to: N. Kaltcheva

1 Tables $1,2,4$ and 5 are only available in electronic form at the CDS via anonymous ftp to 130.79 .128 .5 or via http://cdsweb.u-strasbg.fr/Abstract.html
(1993) have obtained uvby $\beta$ photometry for 16 stars of Car OB2. Shobbrook \& Lynga (1994) collected uvby $\beta$ photometry of 100 early-type stars between $l=288^{\circ}-291.5^{\circ}$, $b=-2^{\circ}-1^{\circ}$ with the aim to investigate the nature of the $\mathrm{OB}$ associations there.

To continue the detailed study of the spatial distribution of the early non-cluster type stars in that direction uvby $\beta$ photometry of 130 stars in the region $l=$ $284^{\circ}-294^{\circ}, b=-3.5^{\circ}-3.5^{\circ}$ is presented in this paper. These stars are brighter than 10 mag and earlier than B4 and are selected from the PPM catalog. About 90 of them are included in the Stephenson \& Sanduleak (1971) catalog. On the sky, they are situated in two areas each with approximate dimensions 4.5 degrees on a side, centered at the Car OB1 and Car OB2 fields. The program stars are plotted in Fig. 1 in Galactic coordinates. The boundaries of Car OB1 and Car OB2 according to the catalog of Humphreys (1978) are also shown.

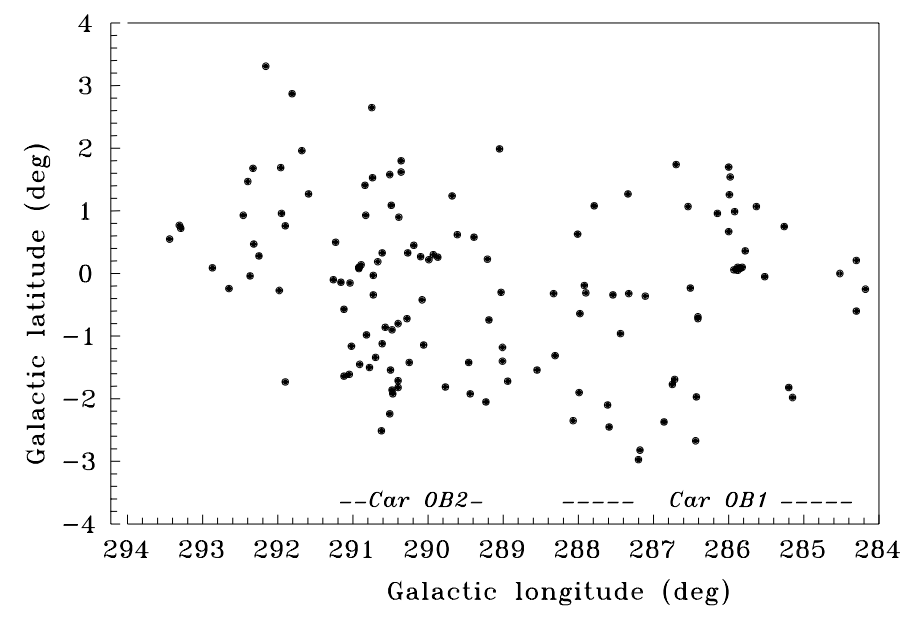

Fig. 1. The program stars plotted in Galactic coordinates 


\section{Observations}

The observations were carried out during the period 7-21 April 1994, using the modular photometer (Kilkenny et al. 1988) on the 0.5-m telescope at the Sutherland site of the South African Astronomical Observatory (SAAO), with a pulse-counting system and a set of SAAO filters. The observations were calibrated to the standard system using standard stars from the lists of Cousins (1987), Perry et al. (1987), Crawford et al. (1971) and Olsen (1983), to cover as large a range as possible of $b-y, m_{1}, c_{1}$ and $\beta$ and to avoid extrapolation in the color equations. The reduction to the standard system was carried out by the procedure adopted at SAAO.

There are two other extensive uvby $\beta$ lists of stars in Carina region. The presented sample contains 18 stars in common with Knude (1992) and 14 with Shobbrook \& Lynga (1994) (hereafter S\&L). Mean differences in $V, b-y$, $m_{1}, c_{1}$ and $\beta$ in the sense (this paper minus others) are shown below (the mean s.e. is of order of 0.001).

\begin{tabular}{llllll}
\hline & $V$ & $b-y$ & $m_{1}$ & $c_{1}$ & $\beta$ \\
\hline Knude & 0.003 & 0.001 & 0.027 & 0.005 & -0.01 \\
S\&L & 0.009 & 0.007 & 0.031 & 0.009 & 0.004 \\
\hline
\end{tabular}

These sets agree quite well in $V, b-y, c_{1}$ and $\beta$ but there is a systematic difference in $m_{1}$. The reduction of the $m_{1}$ values obtained in this paper to the standard system was carried out both using $(v-b)$ and $(b-y)$ colors and $m_{1}$ color differences. The standard stars residuals do not show any systematic differences in both cases. Only a few stars with very negative values of $m_{1}$ are outside the range of the standard stars. For the range of comparison with the lists of Knude (1992) and Shobbrook \& Lynga (1994), the extrapolation in the color equations is fully avoided and any sources of systematic errors in this set of data cannot be seen. This quantity is not used in $E(b-y)$ nor in $M_{v}$ calculations and therefore does not influence the results. Differences of this order in $V, c_{1}$ and $\beta$ leads to an error of $0.2 \mathrm{mag}$ in the $M_{v}$ determination.

The uvby $\beta$ photometry for the program stars is given in Tables 1a (Car OB1 - 50 stars) and 1b (Car OB2 - 80 stars). The stars are referred to these two fields according to their values of galactic longitude $l$.

\section{Reddening and absolute magnitudes}

The parameters derived from the photometry are presented in Tables $2 \mathrm{a}$ and $2 \mathrm{~b}$. The HD numbers, MK types and the reddening-corrected quantities $(b-y)_{0}, m_{0}, c_{0}$ and $V_{0}$ together with the color excess $E(b-y)$ are given in the first six columns. The absolute magnitude $M_{v}$, the true distance moduli $V_{0}-M_{v}$ (for a normal extinction law
$(R=3.2)$ and a coefficient $0.74=E(b-y) / E(B-V))$ and the distance $r$ in parsecs are listed in the last three columns. It is well known that the color excess and absolute magnitude determination via uvby $\beta$ photometric quantities have to be done using different calibrations for the different spectral and luminosity classes. For this reason, special attention was paid to the spectral classification of the program stars. The MK spectral types used here were adopted after a rigorous discussion with W. Buscombe (1994, private communication) on the basis of his collection of MK classification data.

The color excesses for III, IV, V luminosity classes are obtained from the calibration given by Crawford (1978). The intrinsic colors of Kilkenny \& Whittet (1985) are used for the early-type supergiants with values of $c_{0}>-0.17$ and the calibration of Crawford (1978) for values smaller than -0.17 . For this calculation, a simple iteration procedure was adopted using $\left[c_{1}\right]$ as a first value of $c_{0}$.

The absolute magnitude values for all stars are derived via $\beta$. The Balona \& Shobbrook (1984) calibration is used for luminosity classes III, IV, V. In this calibration, attempts are made to correct the main uncertainties in the curve fitting method due to the width of the main sequence and the evolution away from the main sequence of stars at the high-mass end. The errors in the $M_{v}$ values derived in this way are expected to be about $0.2 \mathrm{mag}$. For the supergiants, the $\Delta M_{v} / \Delta \beta$ relation of Zhang (1983) is applied. As is shown in Table IX of Zhang (1983), the standard deviation one should expect applying this calibration is of the same order: 0.2 mag.

It is often questioned whether the distance determination via the values of $\mathrm{H} \beta$ is reliable for O-type stars. There are $24 \mathrm{O}$-type stars in the sample. To compare the results given by the $M_{v} /(u v b y \beta)$ and $M_{v} /$ Sp calibrations, we have calculated the $M_{v}$ values via $M_{v} /$ Sp calibrations of Conti et al. (1983) and have compared them to the values derived via the Balona \& Shobbrook (1984) relation. The results can be seen in Table 3 , where the HD numbers and MK types are listed in the first two columns. The spectroscopic $M_{v}$ values with the corresponding errors, as given in Table 1 of Conti et al. (1983) are shown in the third column. Note that this is the standard deviation of the mean instead of the standard deviation used to estimate the uncertainties concerning the other calibrations discussed here. In the last two columns, the $M_{v}(u v b y \beta)$ values obtained here and the corresponding differences $\left(M_{v} / \mathrm{Sp}-M_{v} /(u v b y \beta)\right)$ are shown for each star. Taking into account the standard errors of the calibrations, these determinations agree within their $1 \sigma$ errors. Only three stars show larger differences which could be due to a wrong LC determination. Deriving the absolute magnitude via $\mathrm{H} \beta$, one should expect that emission in the stellar spectra would make the stars brighter. To avoid this, for all stars with emissions indicated in their spectral classifications, the absolute magnitudes are also estimated via the spectral type - absolute magnitude calibration of Humphreys 

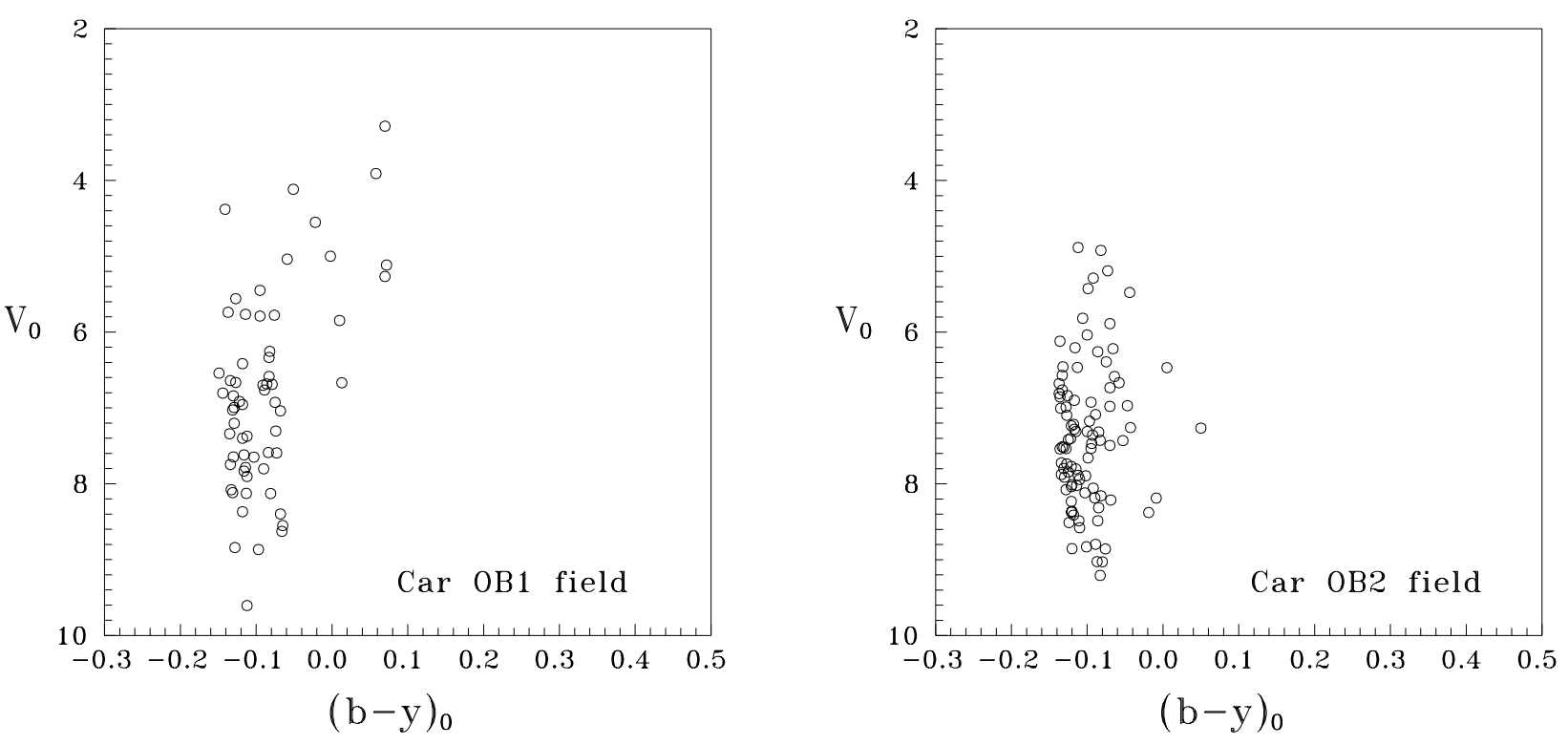

Fig. 2. Color-magnitude diagrams for Car OB1 and Car OB2 fields

Table 3. $M_{v}$-values derived via Spectral types and $\mathrm{H} \beta$ indices for 24 O-type stars. The differences are given in the last column

\begin{tabular}{|c|c|c|c|c|c|}
\hline \multirow[t]{2}{*}{ HD } & \multirow[t]{2}{*}{ MK } & \multicolumn{2}{|c|}{$M_{v}(S p)$} & \multirow[t]{2}{*}{$M_{v}(\beta)$} & \multirow[t]{2}{*}{$\Delta(S p-\beta)$} \\
\hline & & & s.e. & & \\
\hline 90273 & O7Vf & -4.9 & 0.5 & -5.63 & 0.73 \\
\hline 91572 & O7Vf & -4.9 & 0.5 & -5.11 & 0.21 \\
\hline 91598 & O9Ve & -4.4 & 0.6 & -5.28 & 0.88 \\
\hline 92504 & O8Vn & -4.6 & 0.6 & -4.53 & -0.07 \\
\hline 92850 & O9Ib & -6.1 & 0.8 & -5.53 & -0.57 \\
\hline 93403 & O5IIIf & -6.1 & $\ldots$ & -6.16 & 0.06 \\
\hline 94024 & $\mathrm{O} 8 \mathrm{Vn}$ & -4.6 & 0.6 & -5.91 & 1.31 \\
\hline 94370 & O6IIIf & -5.7 & 0.3 & -5.54 & -0.16 \\
\hline 94963 & O6Vf & -5.1 & 0.6 & -5.52 & 0.42 \\
\hline 95589 & O7I & -6.5 & 0.5 & -6.08 & -0.48 \\
\hline 96042 & O9.5Ve & -4.0 & 0.6 & -3.88 & -0.12 \\
\hline 96264 & $\mathrm{O} 6 \mathrm{~V}$ & -5.1 & 0.6 & -4.95 & -0.15 \\
\hline 96715 & $\mathrm{O} 4 \mathrm{Vf}$ & -5.8 & 0.8 & -5.62 & -0.18 \\
\hline 96946 & O7IIIf & -5.6 & 0.6 & -6.02 & 0.42 \\
\hline 97166 & O7.5IIf & -5.0 & 0.4 & -5.52 & 0.52 \\
\hline 97319 & $\mathrm{O} 7.5 \mathrm{~V}$ & -5.0 & $\ldots$ & -4.83 & -0.12 \\
\hline 97399 & O9V & -4.4 & 0.6 & -4.38 & -0.02 \\
\hline 97434 & O7IIIn & -5.6 & 0.6 & -5.86 & 0.26 \\
\hline 97597 & $\mathrm{O} 8 \mathrm{~V}$ & -4.6 & 0.6 & -3.99 & -0.61 \\
\hline 97848 & O7.5II & -5.6 & 0.4 & -4.80 & -0.8 \\
\hline 97913 & $09.5 \mathrm{~V}$ & -4.0 & 0.6 & -5.42 & 1.42 \\
\hline 99160 & O9III & -5.3 & 0.7 & -5.71 & 0.41 \\
\hline 99546 & O7V & -4.9 & 0.5 & -4.88 & -0.02 \\
\hline 305438 & $\mathrm{O} 8 \mathrm{~V}$ & -4.6 & 0.6 & -4.71 & 0.11 \\
\hline
\end{tabular}

\& McElroy (1984) (for B0 - B2 types) and Balona \& Crampton (1974) (for B2.5 - B9 types). The results of these different types of estimation agree well within the errors.

For 14 stars in the fields of Car OB1 and Car OB2 which are earlier than B4-type according to the PPM catalog but were not observed during this run, uvby $\beta$ photometry is available in the literature. For these stars, we have also calculated the reddening-corrected quantities, color excesses, absolute magnitudes and the true distance moduli. These data are shown in Table 4. The third and forth columns of this table contain the MK classification and the sources of the photometric data. Also not included in our sample are thirteen stars referred by Humphreys (1978) to Car OB1 and one star referred to Car OB2. For most of them, $U B V \beta$ photometry is available and the distances can be derived using a $M_{v} / \beta$ calibration. These stars are listed in Table 5 . The photometric data and MK classifications are taken from the OB-star data base of Reed (1993) and the $M_{v}$ values are calculated via the new $M_{v} / \beta$ calibration of Reed \& Beatty (1995).

\section{Results}

The color-magnitude (CM) diagrams for Car OB1 and Car OB2 fields are presented in Fig. 2. All stars from Table 2 of this paper, uvby $\beta$ data for the Car OB2 association (Table 2b, Kaltcheva \& Georgiev 1993) and the stars from Table 4 are included to construct these diagrams. This sample is about $90 \%$ complete concerning the limited magnitude and spectral type mentioned above. There are clearly recognizable main sequences (MS) in both diagrams. Also a presence of evolved stars and presence of foreground stars 

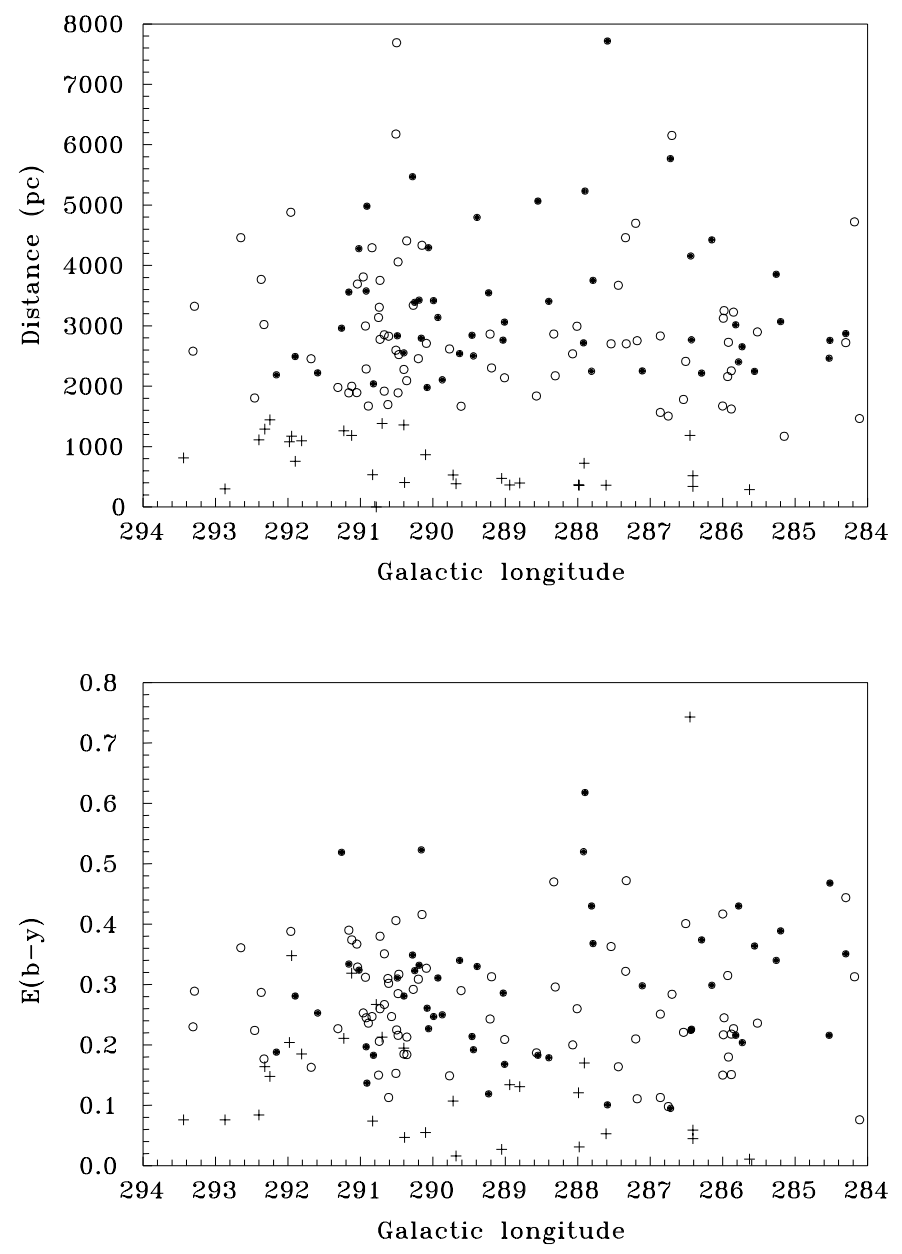

Fig. 3. The obtained distances and color excesses for the program stars as functions of Galactic longitude. The meaning of the symbols used are the same here and for Fig. 4. The stars that form the main sequences in the CM diagrams in the Fig. 2 are marked with "open" symbols. All of them are more distant than $1200 \mathrm{pc}$ in the direction of Car OB1 and $1600 \mathrm{pc}$ in the direction of Car OB2. The stars that are more evolved according to the HR diagrams are marked with "filled" symbols. All of them are more distant than 1600 pc. "+" symbols are used for the stars that are non-MS according to the CM diagrams and low-massive according to the HR diagrams. All of them are nearer than $1600 \mathrm{pc}$

can be seen. The foreground stars should be responsible for the appearance of the "second main sequences" on the CM diagrams, which usually correspond to groups at different distances. It is of interest to see the differences in the spatial distribution between the MS stars and the other stars of the sample.

The derived distances and color excesses for each star are plotted as functions of the Galactic longitude in Fig. 3. The Hertzsprung-Russell (HR) diagrams for the Car OB1 and Car OB2 fields are shown in Fig. 4. To build the HR diagrams, the bolometric corrections from de Jager (1980) are used for the calculation of the luminosities. For
III-V luminosity classes, the effective temperatures were obtained from the relation $T_{\text {eff }} / c_{0}$ of Code et al. (1976). For supergiants, the linear relation between $T_{\text {eff }}$ and $(u-$ $b)_{0}$ obtained from the tables $S p / T_{\text {eff }}$ (Bohm-Vitense 1981) and $(u-b)_{0} / S p$ (Zhang 1983) are used. The theoretical tracks plotted in these figures are from Maeder \& Meynet (1988).

The stars that form the MS in CM diagrams form a very tight main sequence in HR diagrams for both fields. They are marked with "open" symbols in Figs. 3 and 4. According to the distances obtained here, all MS stars are more distant than $1200 \mathrm{pc}$ in the Car OB1 direction and more distant than $1600 \mathrm{pc}$ in the Car OB2 direction. Probably all of them are connected with the Carina Arm. The majority of them are concentrated at $l=286^{\circ}$ and $l=290.7^{\circ}$.

"Filled" and "plus" symbols are used for marking of the non-MS stars. According to the distances obtained, the non MS-stars are both nearby and situated at the Carina Arm. All objects nearer than 1600 pc are low-massive main-sequence objects according to their positions in the HR diagrams. They are marked with "+" symbols in both figures. The distant stars are more evolved and not very massive, according to their positions in the HR diagrams. All of them are more distant than 2100 pc and spread over distances to 6000 pc. They are marked with "filled" symbols.

Figure 3 indicates a link between the Local Arm and the Carina arm at the direction $l=290^{\circ}-293^{\circ}$. The increase of the color excess at this direction seems to be a characteristic of the Local arm.

The stars from Table 5 are not included in the analysis because of the different distance calibration used. For these stars, the obtained distances cover a rather wide interval and the corresponding diagrams remain the same concerning grouping.

\section{Conclusions}

According to the obtained photometry of $\mathrm{O}-\mathrm{B} 4$ stars brighter than $10 \mathrm{mag}$ in the region of the Carina Spiral Feature, the young main-sequence objects are concentrated in narrow intervals of Galactic Longitude at directions $l=286^{\circ}$ and $l=290.7^{\circ}$. The region contains also individual older stars more uniformly spread over the whole region. The value of the color excess $E(B-V)$ for the whole field does not exceed 0.54 mag to a distance of $5 \mathrm{kpc}$.

According to the obtained distances, the observed stars are spread out in depth over more than $3 \mathrm{kpc}$. The mean distance for the more obvious concentration of young stars that appears at $l=290.7^{\circ}$ is $2850 \pm 961 \mathrm{pc}$. The spread one could expect taking into account the uncertainties in $M_{v}(u v b y \beta)$ determination is $\pm 260 \mathrm{pc}$ at this distance. The obtained standard deviation is almost 4 time larger that one could expect if the spread of these stars were not real 


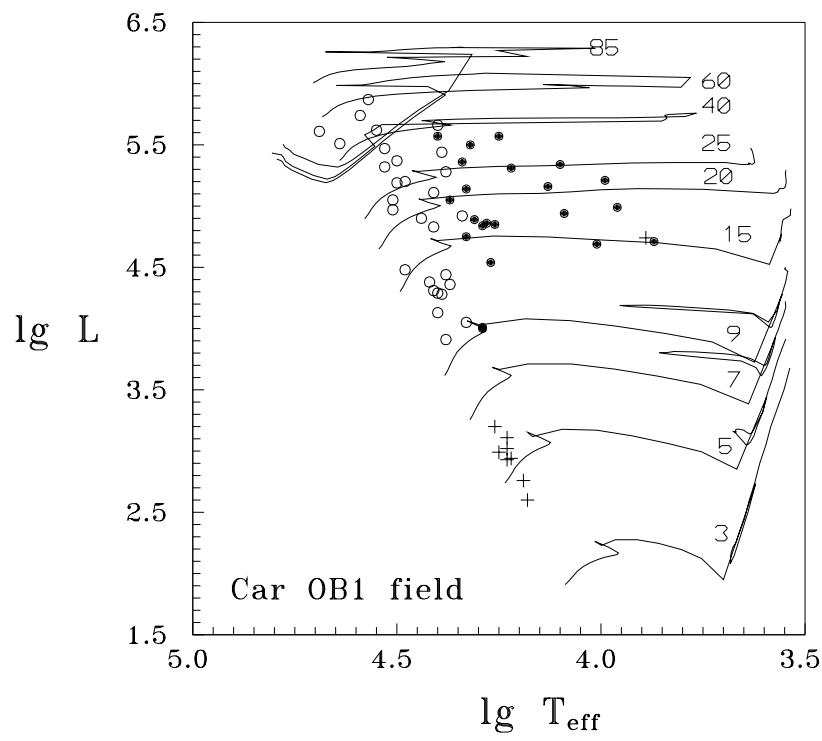

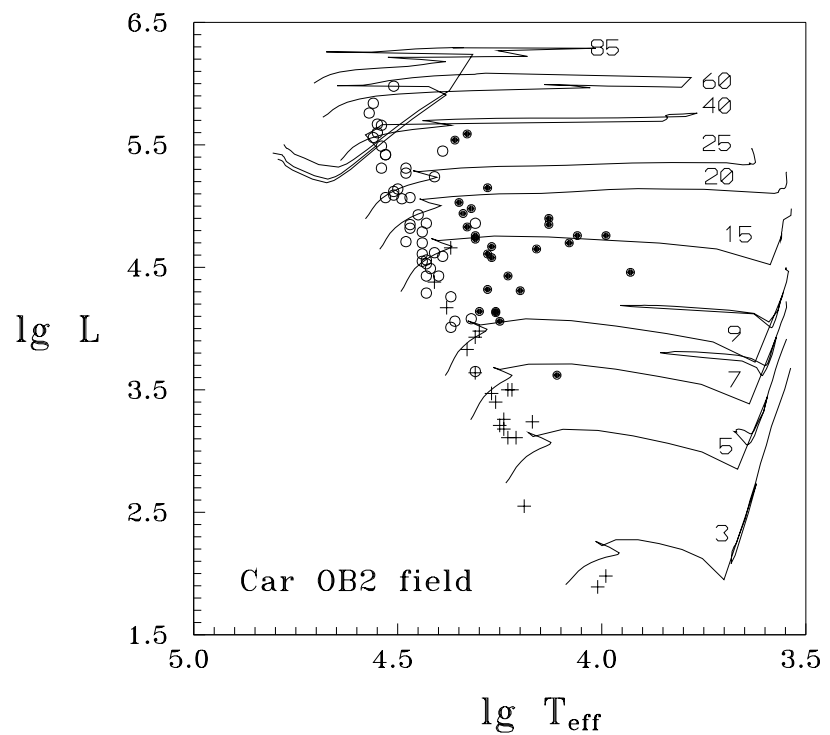

Fig. 4. Hertzsprung-Russell diagrams for Car OB1 and Car OB2 fields. Theoretical tracks are from Maeder \& Meynet (1988). The symbols are the same as for Fig. 3

but due only to errors in distance determination. The $\chi^{2}$ test applied to these stars rejects the null hypothesis of normal distribution of the obtained distances at a $99 \%$ level of significance. The extent of this concentration at a right angle to the line of sight is about $60 \mathrm{pc}$. It is more likely that these stars belong to a huge stellar complex and are seen in projection on the line of sight, rather than representing an association born from a single protostellar cloud. According to this sample, this complex is stretched out over more than $3 \mathrm{kpc}$, while the thickness in the Galactic radial direction is about $200 \mathrm{pc}$ and the thickness in the "Z" direction is $300 \mathrm{pc}$.

According to this sample (Fig. 3), there is a sign of existence an age gradient at the regions approximately $l=284^{\circ}-286^{\circ}$ and $l=289^{\circ}-291^{\circ}$. In these regions, the youngest stars and the more evolved stars do not have exactly the same spatial distribution - the younger stars appear to be closer to the inner part of the Carina Arm in comparison to the more evolved stars. This is more obvious for the field of the Car OB2 complex; for the Car OB1 field the number of the stars observed is not sufficient for even preliminary conclusions.

Acknowledgements. I am grateful to Dr. Dave Kilkenny for his help in the observations and data reduction and to Dr. Cameron Reed for the reading of the manuscript and useful comments. Special thanks are due to Dr. William Buscombe for the valuable discussion of the spectral classification of the stars involved here and to Dr. Wilhelm Seggewiss for comments that significantly improved the last version of the paper. This work was supported by National Bulgarian Science Foundation under contract F 619/1996.

\section{References}

Balona L.A., Crampton D., 1974, MNRAS 166, 203

Balona L.A., Shobbrook R.R., 1984, MNRAS 211

Bohm-Vitense E., 1981, ARA\&A 19, 295

Code A.D., Davis J., Bless R.C., Hanbury Brown R., 1976, ApJ 203, 417

Conti P.S., Garmany C.D., de Loore C., Vanvereren D., 1983, ApJ 274, 302

Cousins A.W.J., 1987, SAAO Circ. 11, 93

Crawford D.L., 1978, AJ 83, 48

Crawford D.L., Barnes J.V., Golson J.C., 1971, AJ 76, 621

de Jager C., 1980, The Brightest Stars. Reidel, Dordrecht

Garcia B., 1994, ApJ 436, 705

Graham J.A., Lynga G., 1965, Mem. Mt Stromlo Observatory, No. 18

Humphreys R.M., 1978, ApJS 38, 309

Humphreys R.M., McElroy D.B., 1984, ApJ 284, 565

Kaltcheva N., Georgiev L., 1993, MNRAS 261, 847

Kilkenny D., Balona L.A., Carter D.B., Ellis D.T., Woodhouse G.F.W., 1988, Mon. Notes Astron. Soc. S. Afr. 47, 69

Kilkenny D., Whittet, 1985, MNRAS 216, 127

Knude J., 1992, A\&ApS 92, 841

Maeder A., Meynet G., 1988, A\&AS 76, 411

Olsen E.H., 1983, A\&AS 54, 55

Perry C.L., Olsen E.H., Crawford D.L., 1987, PASP 99, 1184

Reed C., 1993, PASP 105, 1465

Reed C., Beatty A., 1995, ApJ (in press)

Seggewiss W., 1970, in Proc. IAU Symp. 38, The Spiral Structure of our Galaxy, Becker W., Contopoulos G. (eds.). Reidel, Dordrecht, p. 265

Shobbrook R.R., Lynga G., 1994, MNRAS 269, 857

Stephenson B.C., Sanduleak N., 1971, Warner Swasey Obs. 1, No. 1

Zhang Er.-Ho., 1983, AJ 88, 825 